\title{
Tests of Fundamental Symmetries with Neutrons
}

\author{
B. W. Filippone \\ Kellogg Radiation Laboratory \\ California Institute of Technology \\ Pasadena, CA 91125
}

\begin{abstract}
Because of recent technological developments, new opportunities to test fundamental symmetries using cold and ultra-cold neutrons will become available in the next several years. These tests include studies of the parity-violating hadronic weak interaction, searches for new symmetries beyond the standard model using neutron decay and searches for new sources of Charge-conjugation/Parity (CP) violation through the measurement of the neutron Electric Dipole Moment (EDM).
\end{abstract}

Keywords: Cold Neutrons, Ultra-Cold Neutrons, Symmetries

PACS: 12.15.-y, 12.15.Hh, 29.25.Dz

\section{INTRODUCTION}

Both the observation and violation of fundamental symmetries have played a critical role in the development of the Standard Model of the fundamental interactions. Experiments with free neutrons allow sensitive measurements and tests of a number of symmetries, including parity, Charge conjugation/Parity (CP) and searches for new symmetries beyond the Standard Model. Recent developments in the production of cold and ultra-cold neutrons have provided new opportunities for highly sensitive experiments. I will briefly discuss some new developments in neutron production and summarize the status and plans of existing and future facilities. This will be followed by a summary of the main science objectives within this field and a discussion of the developing program within the US.

\section{NEUTRON TECHNOLOGY}

Very briefly, cold neutrons with wavelengths of approximately $0.4 \mathrm{~nm}$ (with velocities about $100 \mathrm{~m} / \mathrm{s}$ ) are produced from the moderation of fast neutrons generated by either nuclear reactors or proton spallation from a heavy target. These neutrons can then be transported as beams to experiments using neutron guides. The advantages of cold neutrons are their high flux and high polarization using new techniques (polarized ${ }^{3} \mathrm{He}$ and advanced supermirrors) that are under development. Ultra-Cold Neutrons

CP842, Particles and Nuclei,

Seventeenth International Conference on Particles and Nuclei

edited by P. D. Barnes, M. D. Cooper, R. A. Eisenstein, H. van Hecke, and G. J. Stephenson

(C) 2006 American Institute of Physics 0-7354-0338-4/06/\$23.00 
(UCN) are neutrons with wavelengths longer than $50 \mathrm{~nm}$ (with velocities less than about $8 \mathrm{~m} / \mathrm{s}$ ). Neutrons of this velocity can be trapped within certain materials and be highly polarized with modest magnetic fields of $6 \mathrm{~T}$. Materials have been developed that allow trapping of UCN for times on the order of hundreds of seconds. A new source of UCN based on proton spallation and moderation using solid deuterium has been demonstrated recently (Ref. 1). This technique along with a similar technique based on superfluid He may allow significant increases (factors of 100-1000) in the attainable densities of UCN.

\section{FACILITIES FOR FUNDAMENTAL NEUTRON PHYSICS}

Facilities for testing fundamental symmetries with neutrons are typically based at a large neutron scattering facility whose primary mission is based on condensed matter physics and materials science. Such facilities have been constructed at both research reactors and spallation neutron sources. A summary of existing and planned facilities is listed in Table 1.

TABLE 1. Facilities for Fundamental Symmetry Tests with Neutrons

\begin{tabular}{|c|c|c|c|}
\hline Facility & Location & Neutron Technology & Facility Status \\
\hline \multirow[t]{2}{*}{ NG6 } & NIST (US) & Cold Beam & Operating \\
\hline & Reactor & & \\
\hline \multirow{2}{*}{ PF1 \& PF2 } & ILL (France) & Cold Beam and UCN & Operating \\
\hline & Reactor & & \\
\hline \multirow[t]{2}{*}{ FP12 \& Area B } & LANSCE (US) & Cold Beam and UCN & Starting Operations \\
\hline & Spallation source & & \\
\hline \multirow[t]{2}{*}{ FNPB } & SNS (US) & Cold Beam and UCN & Under Construction \\
\hline & Spallation Source & & \\
\hline \multirow[t]{2}{*}{ SINQ } & PSI (Switzerland) & Cold Beam and UCN & Starting Operations \\
\hline & Spallation Source & & \\
\hline \multirow[t]{2}{*}{ FRM-II } & TUM (Germany) & $\mathrm{UCN}$ & Under Construction \\
\hline & Reactor & & \\
\hline \multirow[t]{2}{*}{ PULSTAR } & NCSU (US) & $\mathrm{UCN}$ & Under Construction \\
\hline & Reactor & & \\
\hline \multirow[t]{2}{*}{ LENS } & IUCF (US) & UCN development & Under Construction \\
\hline & Nuclear reaction & & \\
\hline \multirow{2}{*}{ Osaka?, JPARC? } & (Japan) & $\mathrm{UCN}$ & Under Discussion \\
\hline & Spallation Source & & \\
\hline
\end{tabular}

\section{THE HADRONIC WEAK INTERACTION}

While the leptonic weak interactions are well-characterized by the standard electroweak model, purely hadronic weak interactions require a melding of Quantum 
Chromo-Dynamics (QCD) and the electroweak theory. At low energies these processes are non-perturbative and up to now have only been studied in effective theories. These processes are distinguishable from the strong hadronic interactions through the parity violation inherent in the electroweak theory. An early theoretical attempt at estimating the strength of the hadronic weak interactions was undertaken by Desplanques, Donoghue and Holstein (Ref. 2). In this the work the processes could be characterized by six coupling constants: $f_{\pi}, h_{\rho}{ }^{0}, h_{\rho}{ }^{1}, h_{\rho}{ }^{2}, h_{\omega}{ }^{0}, h_{\omega}{ }^{1}$. These couplings were estimated in a quark model and can be best compared with experiment in precision measurements of parity violation in few-body systems. Few-body systems allow comparison with the calculations relatively free of complications from nuclear structure corrections. At present a discrepancy exists in the measurements of the $f_{\pi}$ coupling constant. Nuclear decay measurements in ${ }^{18} \mathrm{~F}$ and atomic parity violation in

${ }^{133} \mathrm{Cs}$ give vastly different results (differing by about an order-of-magnitude). A new measurement of $f_{\pi}$ in polarized neutron capture on protons $(\mathrm{n}+\mathrm{p} \rightarrow \mathrm{d}+\gamma)$ promises to resolve this discrepancy. This experiment is being commissioned at Flight Path 12 (FP12) at the LANSCE facility and is planning to take its high statistics data at the Fundamental Neutron Physics Beamline (FNPB) at the Spallation Neutron Source (SNS) at the Oak Ridge National Laboratory. A new measurement of neutron spin rotation in ${ }^{4} \mathrm{He}$ at the National Institute for Standards and Technology (NIST) will also provide sensitivity to $f_{\pi}$ and several other parameters.

\section{NEUTRON BETA-DECAY}

Neutron beta decay can be described within the Standard Electoweak Model. Precision measurements of the decay properties allow sensitive tests of this model and deviations from the expected decay can be signatures for new physics beyond the Standard Model. An example of constraints on Supersymmetry models from beta decay data is discussed in Ref. 3. In this work the measurements of the CabibboKobayashi-Maskawa matrix element $V_{u d}$ are used to constrain the parameters of the model. Presently the best measurements of $V_{u d}$ are obtained from the decays of radioactive nuclei. Future measurements of neutron decay may eventually provide the best results for $V_{u d}$ because of the potentially smaller uncertainties for the neutron due to nuclear corrections in the case of radioactive nuclei. The decay distribution can be expressed in terms of a total decay rate $\Gamma_{\mathrm{n}}$ and a number of correlation coefficients $b$, $a, A, B, D$ via:

$$
d \Gamma=\Gamma_{n}\left(1+b \frac{m_{e}}{E_{e}}+a \frac{\vec{p}_{e} \cdot \vec{p}_{v}}{E_{e} E_{v}}+A \frac{\vec{\sigma}_{n} \cdot \vec{p}_{e}}{E_{e}}+B \frac{\vec{\sigma}_{n} \cdot \vec{p}_{v}}{E_{v}}+D \frac{\vec{\sigma}_{n} \cdot\left(\vec{p}_{e} \times \vec{p}_{v}\right)}{E_{e} E_{v}}\right)
$$

Coefficients $b$ and $D$ are zero in the Standard Model, while the other coefficients depend, in different ways, on other parameters. The dependence of neutron decay on $V_{u d}$ can be seen in the expression for the total decay rate: 


$$
\frac{1}{\tilde{\mathrm{A}}_{\mathrm{n}}}=\left(\frac{2 \partial^{3} \hbar^{7}}{\mathrm{~m}_{\mathrm{e}}^{5} \mathrm{c}^{4}}\right)\left(\frac{1}{\mathrm{G}_{\mathrm{F}}^{2}\left|\mathrm{~V}_{\mathrm{ud}}\right|^{2}}\right)\left[\frac{1}{1+3\left(\mathrm{G}_{\mathrm{A}} / \mathrm{G}_{\mathrm{V}}\right)^{2}}\right] \frac{1}{\mathrm{f}\left(1+\ddot{\mathrm{A}}_{\mathrm{R}}\right)}
$$

where $G_{F}$ is the Fermi constant which is known from muon decay, $V_{u d}$ is the updown quark weak coupling from the Cabibbo-Kobayashi-Maskawa (CKM) matrix. $G_{A} / G_{V}$ is the ratio of the axial vector to vector weak coupling constant, $f$ is the phase space integral for the decay products and $\Delta_{R}$ is an electroweak radiative correction.

Extraction of $V_{u d}$ using the neutron requires measurement of the neutron lifetime $\left(\tau_{\mathbf{n}}=\right.$ $1 / \Gamma_{\mathrm{n}}$ ) and a separate measurement of $G_{A} / G_{V}$. The measurement of $G_{A} / G_{V}$ can be accomplished by a measurement of the $A$ correlation from eq. (1). Within the Standard Model, and to leading order, the $A$ correlation is related to $G_{A} / G_{V}$ via:

$$
\mathbf{A}=\frac{-2 \ddot{\mathbf{e}}(1+\ddot{\mathbf{e}})}{1+3 \ddot{\mathrm{e}}^{2}} \quad, \ddot{\mathrm{e}}=\frac{G_{A}}{G_{V}}
$$

The present status of measurements of $V_{u d}$ from neutron decay is shown in Fig. 1.

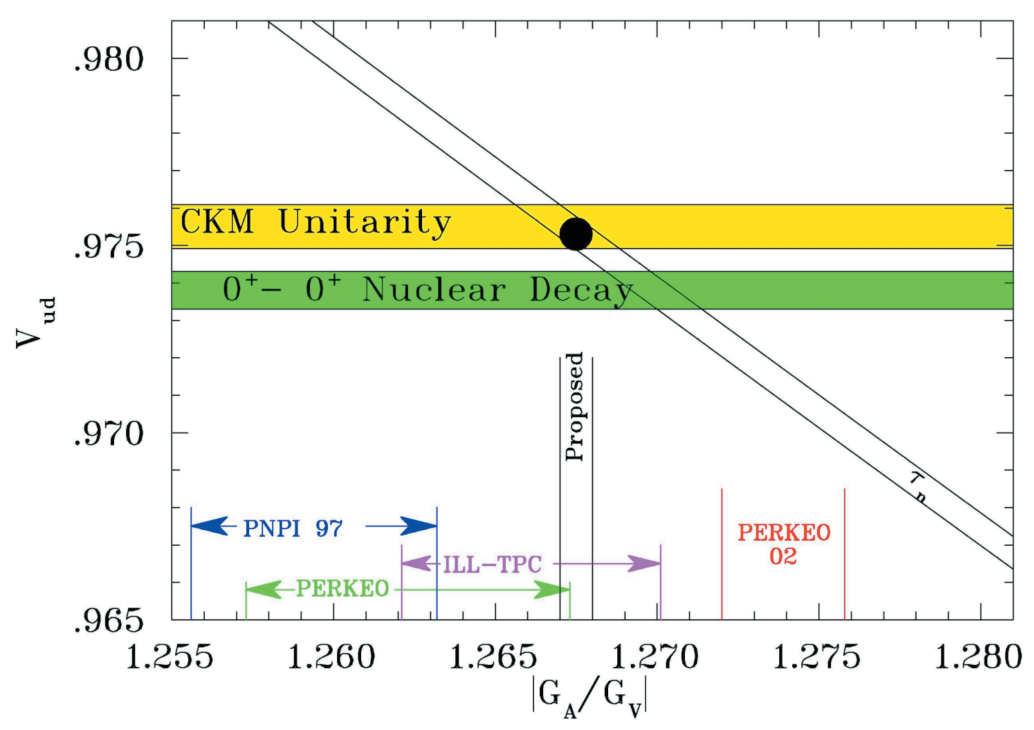

FIGURE 1. Status of $V_{u d}$ in terms of the neutron lifetime and neutron decay asymmetry measurements based on data from Ref. 4. The solid circle is the proposed measurement at the LANSCE facility in Area B. 
While improved measurements of $G_{A} / G_{V}$ from the $A$ correlation coefficient are planned at the UCNA experiment at the LANSCE facility and at the FNPB, a recent measurement of the neutron lifetime has suggested that the value of this important quantity is still unresolved. A summary of recent measurements of the neutron lifetime is shown in Fig. 2, where the most recent measurement deviates from the previous average by about six standard deviations. Clearly new measurements of this quantity are necessary in order to resolve this serious discrepancy.

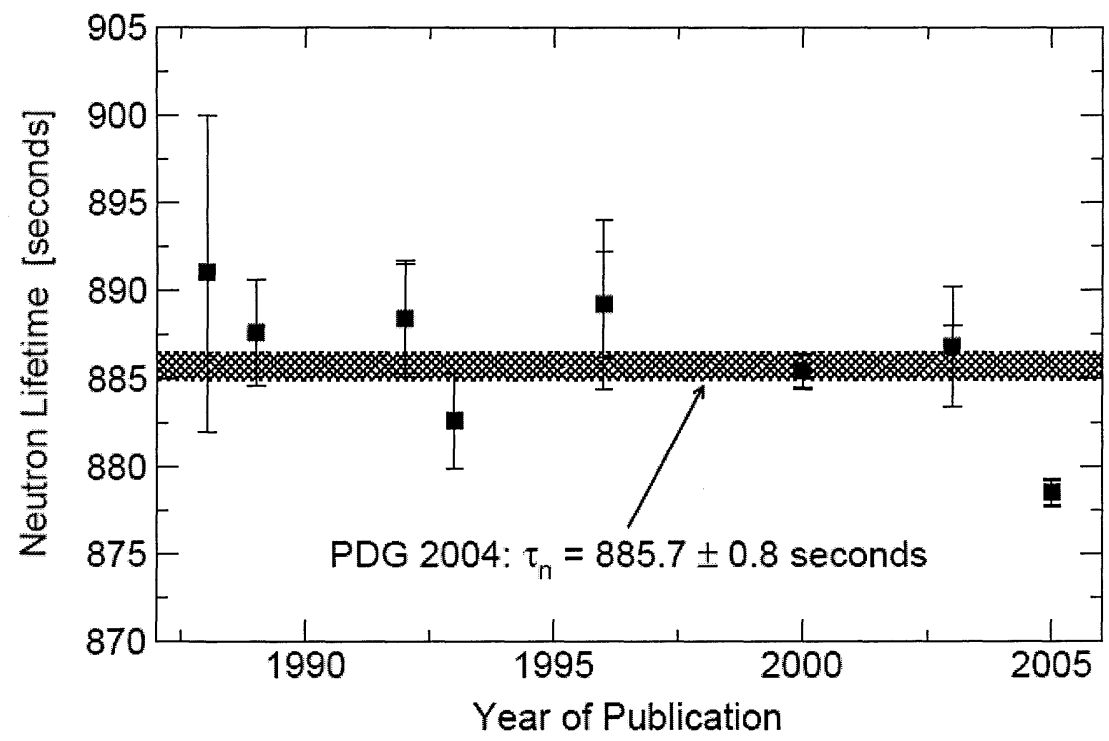

FIGURE 2. Recent measurements of the neutron lifetime. Both cold neutron beams and ultra-cold neutron bottles were used in these experiments. The most recent measurement (Ref. 5) differs from the mean value of the previous measurements by about six standard deviations.

\section{THE NEUTRON ELECTRIC DIPOLE MOMENT (EDM)}

The observation of a non-zero neutron Electric Dipole moment (EDM) would provide the first evidence for Time-Reversal violation (and, hence, $\mathrm{CP}$ violation via the CPT theorem) in the flavor-conserving sector of the Standard Model. This type of $\mathrm{CP}$ violation in baryons may provide an explanation for the matter-antimatter asymmetry observed in the universe.

For more than 50 years, searches for a neutron EDM have yielded no observable signal, even as the sensitivity has increased by eight orders-of-magnitude. The current best upper limit of $6.3 \times 10^{-26} \mathrm{e}-\mathrm{cm}$ (at $90 \%$ C.L.) (Ref. 6) is about six orders-ofmagnitude above the Standard Model prediction. Thus there exists a significant opportunity for observing new physics in searches with improved sensitivity. Some of the proposed extensions to the standard model (e.g., Supersymmetry) predict a value 
for the neutron electric dipole moment that is only one or two orders of magnitude below the present limit. A new search then has the potential to severely constrain extensions to the standard model or, if a non-zero value is observed, to provide the first evidence for a neutron electric dipole moment at a level larger than that of the standard model prediction. Such a measurement of a neutron EDM would indicate the existence of a new source of CP violation not currently included in the Standard Model.

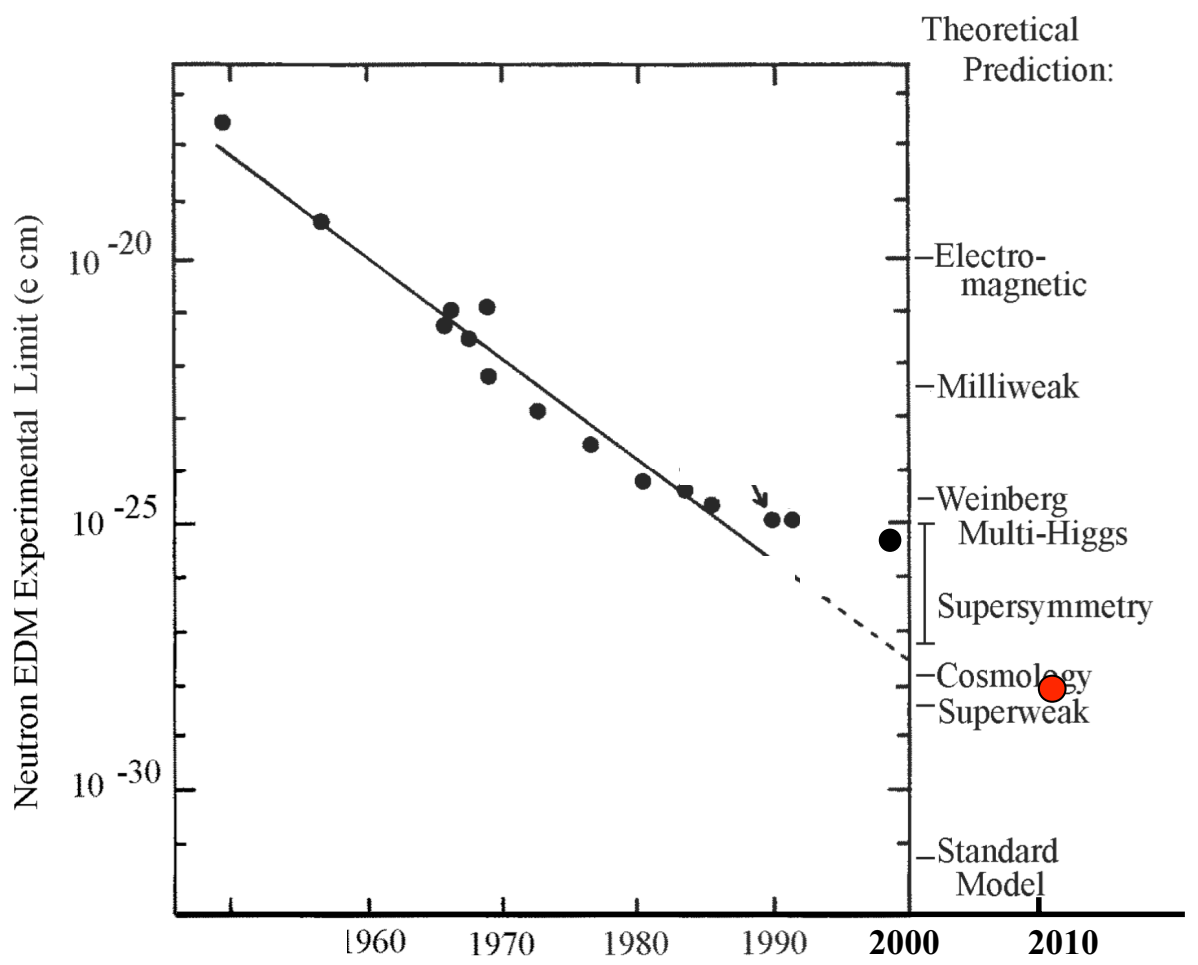

FIGURE 3. Time dependence of the measured upper limit for the neutron EDM. The red circle represents the expected sensitivity from the EDM measurement at the FNPB as described in the text.

Several new measurements of the neutron EDM are being planned including one to be performed at the FNPB at the SNS. This new search will employ ultra-cold neutrons produced via the moderation of cold neutrons in superfluid ${ }^{4} \mathrm{He}$ (with the recoil phonon in the superfluid carrying away the missing momentum and energy). The experiment is based on the magnetic resonance technique of free precession of a magnetic moment in a magnetic field and will also use a novel polarized ${ }^{3} \mathrm{He}$ comagnetometer. The value of the electric dipole moment can be extracted from the 
difference between neutron spin precession frequencies under the influence of parallel and anti-parallel magnetic and electric fields. The proposed sensitivity of the new experiment can be seen in Fig. 3, where the previous upper limits on the EDM are shown vs. the date of the published result. A comparison of the sensitivity of the proposed SNS experiment to the present experiment at ILL is shown in Table. 2. The sensitivity is calculated using:

$$
\dot{o}_{\mathrm{d}} \cong \frac{\hbar}{4|\vec{E}| T_{m} \sqrt{\mathrm{mN}_{\mathrm{UCN}}}}
$$

TABLE 2. Comparison of EDM experiments

\begin{tabular}{lll}
\hline Experimental Parameter & \multicolumn{1}{c}{ Present ILL experiment } & Proposed SNS experiement \\
\hline$N_{U C N}$ & $1.3 \times 10^{4}$ & $2 \times 10^{7}$ \\
$|\mathrm{E}|(\mathrm{kV} / \mathrm{cm})$ & $4.5 \mathrm{kV} / \mathrm{cm}$ & $50 \mathrm{kV} / \mathrm{cm}$ \\
$\mathrm{T}_{\mathrm{m}}(\mathrm{sec})$ & 140 & 500 \\
$\mathrm{M}(\mathrm{cycles} /$ day $)$ & 270 & 50 \\
$\sigma_{\mathrm{d}}(\mathrm{e}-\mathrm{cm}) /$ day & $6 \times 10^{-25}$ & $1.5 \times 10^{-27}$ \\
\hline
\end{tabular}

\section{OUTLOOK}

New tests of fundamental symmetries using neutrons are underway both in the US and worldwide. Compelling science is anticipated from these studies within the next 510 years in measurements of the nucleon-nucleon weak current, neutron beta decay and the neutron EDM.

\section{ACKNOWLEDGMENTS}

The author acknowledges support from the National Science Foundation through grant 0244899.

\section{REFERENCES}

1. A. Saunders, et al., Phys. Lett. B 593, 55-60 (2004).

2. B. Desplanques, J. F. Donoghue, B. R. Holstein, Annals of Physics 124, 449-495 (1980).

3. A. Kurylov and M.J. Ramsey-Musolf, Phy. Rev. Lett. 88, 071804 (2002).

4. Particle Data Group, S. Eidelman, et al., Phys. Lett. B 592, 1-1109 (2004).

5. A. Serebrov, et al., Phys. Lett. B 605, 72-28 (2005).

6. P. G. Harris, et al., Phys. Rev. Lett. 82, 904-907 (1999). 